\title{
Two numerical iteration methods for solving absolute value equations
}

\author{
Jun He*, Yanmin Liu, Junkang Tian \\ School of Mathematics, Zunyi Normal College, Zunyi, Guizhou, 563006, China \\ *Corresponding author, e-mail: hejunfan1@163.com
}

Received 8 Jun 2017

Accepted 16 Dec 2017

\begin{abstract}
We introduce two kinds of iteration methods based on the generalization of the hermitian and skewhermitian splitting. Their convergence properties are established. We show the effectiveness of our methods.
\end{abstract}

KEYWORDS: linear complementarity problem; GHSS-based iteration; Picard-HSS iteration; nonlinear HSS-like iteration

MSC2010: 65F12 65L05 65N22

\section{INTRODUCTION}

We consider the following absolute value equation (AVE):

$$
A x-|x|=b, \quad A \in \mathbb{C}^{n \times n}, \quad x, b \in \mathbb{C}^{n},
$$

where $|x|$ denotes the componentwise absolute value of the vector $x$. The system (1), which is generally equivalent to the linear complementarity problem ${ }^{1,2}$, arises from linear programming, quadratic programming, and other engineering problems $^{3,4}$.

In the last decade, some numerical methods have been developed to solve AVEs. These include the SLP method ${ }^{5}$, the semismooth Newton method and its inexact variants ${ }^{6-8}$, the sign ac$\operatorname{cord}_{\text {method }}{ }^{9}$, the hybrid algorithm ${ }^{10}$, the PicardCSCS iteration method, and the nonlinear CSCSlike iteration method ${ }^{11}$. When $A \in \mathbb{C}^{n \times n}$ is a nonHermitian positive definite matrix, the Hermitian and skew-Hermitian splitting (HSS) iteration was first introduced by Bai et $\mathrm{al}^{12}$ and extended in Ref. 13 for the solution of a class of non-Hermitian linear systems $A x=b$. Based on the Hermitian and skew-Hermitian splitting, the Picard-HSS iteration method, nonlinear HSS-like iteration method, and the relaxed nonlinear PHSS-like iteration method are introduced to solve AVEs ${ }^{14,15}$.

In this paper, two kinds of GHSS-based iteration methods are established to solve (1) efficiently. These are based on the following generalization of the hermitian and skew-hermitian splitting (GHSS):

$$
A=G+K+S
$$

where $H=\frac{1}{2}\left(A+A^{\mathrm{T}}\right)=G+K, G$ and $K$ are Hermitian positive semidefinite, and $S=\frac{1}{2}\left(A-A^{\mathrm{T}}\right)$. Firstly, convergence conditions of the Picard-GHSS iterative method will be investigated. Then the convergence condition of the nonlinear GHSS-like iteration method is proved by Zhang's techniques ${ }^{15}$.

The reminder of this paper is organized as follows. First, we introduce several preliminary results concerning nonsmooth analysis. Then we introduce the Picard-GHSS and the nonlinear GHSSlike iterative methods to solve (1) and investigate its convergence properties. Finally, numerical experiments are reported to show the effectiveness of our methods.

\section{PRELIMINARIES}

Let $\Psi: \mathbb{R}^{n} \rightarrow \mathbb{R}^{n}$ be a specified function, and let $x$ be a given point in $\mathbb{R}^{n}$. The function $\Psi$ is supposed to be locally Lipschitzian near $x$ if there exist a scalar $\kappa \in \mathbb{R}$ and $\delta>0$ such that for all $y, z \in \mathbb{R}^{n},\|y-x\|<$ $\delta$, $\|z-x\|<\delta$, the following inequality holds:

$$
\|\Psi(y)-\Psi(z)\|<\kappa\|y-z\| .
$$

Let $\Psi: \mathbb{R}^{n} \rightarrow \mathbb{R}^{n}$ be a locally Lipschitzian function. From Rademacher's theorem ${ }^{15}, \Psi$ is differentiable almost everywhere. Denote the set of points at which $\Psi$ is differentiable by $D_{\Psi}$. We write $\Psi^{\prime}(x)$ for the usual $n \times n$ Jacobian matrix of partial derivatives whenever $x$ is a point at which the necessary partial derivatives exist. Then, the Bouligand subdifferential of $\Psi$ at $x \in \mathbb{R}^{n}$, denoted by $\partial_{\mathrm{B}} \Psi(x)$, is given by

$\partial_{\mathrm{B}} \Psi(x):=\left\{\lim _{k \rightarrow \infty} \Psi^{\prime}(x)\left(x^{(k)}\right): x^{(k)} \in D_{\Psi}, x^{(k)} \rightarrow x\right\}$. 
Clarke's generalized Jacobian ${ }^{16}$ of $\Psi$ at $x$ is the convex hull of $\partial_{\mathrm{B}} \Psi(x)$, i.e.,

$$
\partial \Psi(x)=\operatorname{conv}\left\{\partial_{\mathrm{B}} \Psi(x)\right\} .
$$

Since $\Psi$ is a locally Lipschitzian function, the set $\partial_{\mathrm{B}} \Psi(x)$ and $\partial \Psi(x)$ are bounded. By the definition, $\partial_{\mathrm{B}} \Psi(x)$ is also closed. So $\partial_{\mathrm{B}} \Psi(x)$ and $\partial \Psi(x)$ are compact.

Definition 1 [Ref. 17] $\Psi$ is called semismooth at $x$ if $\Psi$ is locally Lipschitzian and for all $h \in \mathbb{R}$. with $h \neq 0$, and

$$
\lim _{h^{\prime} \rightarrow h, t \downarrow 0}\left\{V h^{\prime}: V \in \partial \Psi\left(x+t h^{\prime}\right)\right\}
$$

exists. If $\Psi$ is semismooth at all points in a given set, we say that $\Psi$ is semismooth in this set.

If $\Psi$ is semismooth at $x$, then $\Psi$ must be directionally differentiable at $x$.

Proposition 1 (Refs. 17, 18) Suppose that $\Psi$ is semismooth at $x$. Then the classic directional derivative

$$
\Psi^{\prime}(x ; h)=\lim _{t \downarrow 0} \frac{\Psi(x+t h)-\Psi(x)}{t}
$$

exists and is equal to the limit in (2).

\section{THE PICARD-GHSS ITERATIVE METHOD}

Motivated by Ref. 13, we give the following two numerical iteration methods to solve (1).

Algorithm 1 (Picard-GHSS iterative method) Let $A \in \mathbb{C}^{n \times n}$ be a sparse and positive definite matrix, and $H=\frac{1}{2}\left(A+A^{\mathrm{T}}\right)=G+K$ and $S=\frac{1}{2}\left(A-A^{\mathrm{T}}\right)$ be its Hermitian and skew-Hermitian parts, respectively. Given an initial guess $x^{(0)} \in \mathbb{C}^{n}$, compute $x^{(k+1)}$ for $k=0,1,2, \ldots$ using the following iteration scheme until $\left\{x^{(k)}\right\}$ satisfies the stopping criterion:

$$
\begin{aligned}
(\alpha I+G) x^{(k+1 / 2)} & =(\alpha I-S-K) x^{(k)}+\left|x^{(k)}\right|+b, \\
(\alpha I+S+K) x^{(k+1)} & =(\alpha I-G) x^{(k+1 / 2)}+\left|x^{(k)}\right|+b,
\end{aligned}
$$

where $\alpha$ is a given positive constant and $I$ is the identity matrix.

Let $\mathscr{M}(\alpha)=(\alpha I+S+K)^{-1}(\alpha I-G)(\alpha I+$ $G)^{-1}(\alpha I-S-K), \mathscr{G}(\alpha)=2 \alpha(\alpha I+S+K)^{-1}(\alpha I+$ $G)^{-1}$, where $\alpha$ is a positive constant and $I$ is the identity matrix of order $n$. The following theorem suggests sufficient conditions for the convergence of the Picard-GHSS iteration method for solving (1).
Theorem 1 Let $A \in \mathbb{C}^{n \times n}$ be a sparse and positive definite matrix, $H=\frac{1}{2}\left(A+A^{\mathrm{T}}\right)=G+K$ and $S=$ $\frac{1}{2}\left(A-A^{\mathrm{T}}\right)$ be its Hermitian and skew-Hermitian parts, respectively. Let also $\eta=\left\|A^{-1}\right\|_{2}<1$. Then (1) has a unique solution $x^{*}$, and for any initial guess $x^{(0)} \in \mathbb{C}^{n}$ and any sequence of positive integers $\ell_{k}, k=$ $0,1,2, \ldots$, the iteration sequence $\left\{x^{(k)}\right\}_{k=0}^{\infty}$ produced by the Picard-GHSS iteration method converges to $x^{*}$ provided that $l=\liminf _{k \rightarrow \infty} l_{k} \geqslant N$, where $N$ is a natural number satisfying

$$
\left\|(\mathscr{M}(\alpha))^{s}\right\|_{2}<\frac{1-\eta}{1+\eta}, \quad \forall s \geqslant N .
$$

Proof: Since $\eta<1$ and from the conclusion of Ref. 19, AVE (1) has a unique solution $x^{*} \in \mathbb{C}^{n}$. As with the proof of the sufficient condition for the convergence of the Picard-HSS iteration method ${ }^{15}$, for $k=0,1,2, \ldots$, the $(k+1)$ th iterate of the PicardGHSS iteration is

$x^{(k+1)}=(\mathscr{M}(\alpha))^{l_{k}} x^{(k)}+\sum_{j=0}^{l_{k}-1}(\mathscr{M}(\alpha))^{j} \mathscr{G}(\alpha)\left(\left|x^{(k)}\right|+b\right)$.

On the other hand, since $x^{*}$ is the solution of (1), we can obtain

$$
x^{*}=(\mathscr{M}(\alpha))^{l_{k}} x^{*}+\sum_{j=0}^{l_{k}-1}(\mathscr{M}(\alpha))^{j} \mathscr{G}(\alpha)\left(\left|x^{*}\right|+b\right) .
$$

Subtracting (4) from (3) yields

$$
\begin{aligned}
x^{(k+1)}-x^{*}= & (\mathscr{M}(\alpha))^{l_{k}}\left(x^{(k)}-x^{*}\right) \\
& +\sum_{j=0}^{l_{k}-1}(\mathscr{M}(\alpha))^{j} \mathscr{G}(\alpha)\left(\left|x^{(k)}\right|-\left|x^{*}\right|\right) .
\end{aligned}
$$

Furthermore, since $\rho(\mathscr{M}(\alpha))<1$, we obtain

$$
\begin{aligned}
& \sum_{j=0}^{l_{k}-1}(\mathscr{M}(\alpha))^{j} \mathscr{G}(\alpha) \\
& \quad=\left(I-(\mathscr{M}(\alpha))^{l_{k}}\right)(I-\mathscr{M}(\alpha))^{-1} \mathscr{G}(\alpha) \\
& \quad=\left(I-(\mathscr{M}(\alpha))^{l_{k}}\right) A^{-1} .
\end{aligned}
$$

Substituting the above identity in (5) yields

$$
\begin{aligned}
& x^{(k+1)}- x^{*} \\
&=\mathscr{M}(\alpha))^{l_{k}}\left(x^{(k)}-x^{*}\right) \\
& \quad+\left(I-(\mathscr{M}(\alpha))^{l_{k}}\right) A^{-1}\left(\left|x^{(k)}\right|-\left|x^{*}\right|\right) \\
&=(\mathscr{M}(\alpha))^{l_{k}}\left[\left(x^{(k)}-x^{*}\right)-A^{-1}\left(\left|x^{(k)}\right|-\left|x^{*}\right|\right)\right] \\
& \quad+A^{-1}\left(\left|x^{(k)}\right|-\left|x^{*}\right|\right) .
\end{aligned}
$$


Hence

$\left\|x^{(k+1)}-x^{*}\right\|_{2} \leqslant\left(\left\|(\mathscr{M}(\alpha))^{l_{k}}\right\|_{2}(1+\eta)+\eta\right)\left\|x^{(k)}-x^{*}\right\|_{2}$

The above inequality is true due to the fact that for any $x, y \in \mathbb{C}^{n}$. It follows that $\||x|-|y|\|_{2} \leqslant\|x-y\|_{2}$. Since $\rho(\mathscr{M}(\alpha))<1$, we have $\lim _{s \rightarrow \infty}(\mathscr{M}(\alpha))^{s}=0$. Thus there exists a natural number $N$ such that

$$
\left\|(\mathscr{M}(\alpha))^{s}\right\|_{2}<\frac{1-\eta}{1+\eta}, \quad \forall s \geqslant N .
$$

If we suppose that $l=\liminf _{k \rightarrow \infty} l_{k} \geqslant N$.

\section{THE NONLINEAR GHSS-LIKE ITERATIVE METHOD}

Algorithm 2 (Nonlinear GHSS-like iterative method) Let $A \in C^{n \times n}$ be a sparse and positive definite matrix, and $H=\frac{1}{2}\left(A+A^{\mathrm{T}}\right)=G+K$ and $S=\frac{1}{2}\left(A-A^{\mathrm{T}}\right)$ be its Hermitian and skew-Hermitian parts, respectively. Given an initial guess $x^{(0)} \in C^{n}$, compute $x^{(k+1)}$ for $k=0,1,2, \ldots$ using the following iteration scheme until $\left\{x^{(k)}\right\}$ satisfies the stopping criterion:

$$
\begin{aligned}
(\alpha I+G) x^{(k+1 / 2)}= & (\alpha I-S-K) x^{(k)}+\left|x^{(k)}\right|+b, \\
(\alpha I+S+K) x^{(k+1)}=( & \alpha I-G) x^{(k+1 / 2)} \\
& +\left|x^{(k+1 / 2)}\right|+b,
\end{aligned}
$$

where $\alpha$ is a given positive constant and $I$ is the identity matrix.

Define

$$
\begin{aligned}
& \mathscr{U}(x)=(\alpha I+G)^{-1}[(\alpha I-S-K) x+|x|+b], \\
& \mathscr{V}(x)=(\alpha I+S+K)^{-1}[(\alpha I-G) x+|x|+b],
\end{aligned}
$$

and

$$
\Theta(x)=\mathscr{V} \circ \mathscr{U}(x):=\mathscr{V}(\mathscr{U}(x)) .
$$

Then the nonlinear GHSS-like iterative scheme can be equivalently expressed as

$$
x^{(k+1)}=\Theta\left(x^{(k)}\right) \text {. }
$$

Using Zhang's techniques ${ }^{15}$, we will analyse the convergence of the nonlinear GHSS-like iteration method.

Definition 2 [Ref. 20] Let $\Theta: \mathbb{D} \subset \mathbb{R}^{n} \rightarrow \mathbb{R}^{n}$. Then $x^{*}$ is a point of attraction of the iteration (8) if there is an open neighbourhood $S$ of the point $x^{*}$ such that $S \subset \mathbb{D}$ and, for any $x^{(0)} \in S$, the iterates $\left\{x^{(k)}\right\}$ all lie in $\mathbb{D}$ and converge to $x^{*}$.
Proposition 2 (Ref. 15) Suppose that $\Theta: \mathbb{R}^{n} \rightarrow \mathbb{R}^{n}$ has a fixed point $x^{*} \in \mathbb{R}^{n}$ and is semismooth at $x^{*}$. If for all $V \in \partial_{\mathrm{B}} \Theta\left(x^{*}\right)$, we have $\rho(V)<1$, where $\rho(V)$ denotes the spectral radius of $V$. Then $x^{*}$ is a point of attraction of the iteration scheme (8).

From statements in Ref. 15 , let $x^{*}$ satisfy $A x^{*}-$ $\left|x^{*}\right|=b$. We compute the Bouligand subdifferential of $\Theta\left(x^{*}\right)$ defined by (7)-(8) at $x^{*}$. Because of the special form of $\mathscr{U}$ and $\mathscr{V}$, it is easy to verify that, $x^{*}=\mathscr{U}\left(x^{*}\right), x^{*}=\mathscr{V}\left(x^{*}\right)$, and $x^{*}=\Theta\left(x^{*}\right)$. Observe the special form of $\Theta$. We have that

$$
\begin{aligned}
\partial_{\mathrm{B}} \Theta\left(x^{*}\right)= & \left\{\lim _{k \rightarrow \infty} \Theta^{\prime}\left(x^{(k)}\right): x^{(k)} \in D_{\Theta}, x^{(k)} \rightarrow x^{*}\right\} \\
= & \left\{\lim _{k \rightarrow \infty} \mathscr{V}^{\prime}\left(y^{(k)}\right) \mathscr{U}^{\prime}\left(x^{(k)}\right): x^{(k)} \in D_{\mathscr{U}},\right. \\
& \left.y^{(k)}=\mathscr{V}\left(x^{(k)}\right) \in D_{\mathscr{V}}, x^{(k)} \rightarrow x^{*}\right\} \\
\subset & \left\{\lim _{y^{(k) \rightarrow x^{*}}} \mathscr{V}^{\prime}\left(y^{(k)}\right): y^{(k)} \in D_{\mathscr{V}}\right\},
\end{aligned}
$$

and

$$
\begin{aligned}
\left\{\lim _{x^{(k) \rightarrow x^{*}}} \mathscr{U}^{\prime}\left(x^{(k)}\right): x^{(k)} \in\right. & \left.D_{\mathscr{U}}\right\} \\
& \subset \partial_{\mathrm{B}} \mathscr{V}\left(x^{*}\right) \partial_{\mathrm{B}} \mathscr{U}\left(x^{*}\right),
\end{aligned}
$$

where

$$
\begin{aligned}
\partial_{\mathrm{B}} \mathscr{V}\left(x^{*}\right) \partial_{\mathrm{B}} \mathscr{U}\left(x^{*}\right): & =\{W: W=E F, \\
E & \left.\in \partial_{\mathrm{B}} \mathscr{V}\left(x^{*}\right), F \in \partial_{\mathrm{B}} \mathscr{U}\left(x^{*}\right)\right\} .
\end{aligned}
$$

According to the above discussion and Proposition 2 , we immediately obtain the following conclusion about the convergence of the nonlinear GHSSlike iteration method.

Theorem 2 Let the point $x^{*}$ satisfy $A x^{*}=\left|x^{*}\right|+b$. Let $A \in C^{n \times n}$ be a sparse and positive definite matrix, and $H=\frac{1}{2}\left(A+A^{\mathrm{T}}\right)=G+K$ and $S=\frac{1}{2}\left(A-A^{\mathrm{T}}\right)$ be its Hermitian and skew-Hermitian parts, respectively. Furthermore, $F, \tilde{F} \in \partial_{\mathrm{B}}\left|x^{*}\right|$. We write

$$
\mathscr{M}(\alpha ; F, \tilde{F})=\mathscr{T}_{1}(\alpha ; F) \mathscr{T}_{2}(\alpha ; \tilde{F}),
$$

where

$$
\begin{aligned}
& \mathscr{T}_{1}(\alpha ; F)=(\alpha I+S+K)^{-1}[(\alpha I-G)+F], \\
& \mathscr{T}_{2}(\alpha ; \tilde{F})=(\alpha I+G)^{-1}[(\alpha I-S-K)+\tilde{F}] .
\end{aligned}
$$

If for all $F, \tilde{F} \in \partial_{\mathrm{B}}\left|x^{*}\right|, \rho(\mathscr{M}(\alpha ; F, \tilde{F}))<1$, then $x^{*}$ is a point of attraction of the nonlinear GHSS-like iteration method. 
Proof: It is clear that $\mathscr{U}$ and $\mathscr{V}$ are semismooth and so $\Theta$ is semismooth. Let $E \in \partial_{\mathrm{B}}\left|x^{*}\right|$. Then it is not hard to show that $E$ is a diagonal matrix:

$$
E=\operatorname{diag}\left(E_{11}, E_{22}, \ldots, E_{n n}\right) .
$$

We have $E_{i i}=1$, if $x_{i}^{*}>0 ; E_{i i}=-1$, if $x_{i}^{*}<0$, and $E_{i i} \in\{1,-1\}$, if $x_{i}^{*}=0$. If $W \in \partial_{\mathrm{B}} \mathscr{F}\left(x^{*}\right)$, then $W=(\alpha I+S+K)^{-1}[(\alpha I-G)+F]$, where $F \in \partial_{\mathrm{B}}\left|x^{*}\right|$. If $\tilde{W} \in \partial_{\mathrm{B}} \mathscr{U}\left(x^{*}\right)$, then $\tilde{W}=(\alpha I+$ $G)^{-1}[(\alpha I-S-H)+\tilde{F}]$, where $\tilde{F} \in \partial_{\mathrm{B}}\left|x^{*}\right|$. Since $\partial_{\mathrm{B}} \Theta\left(x^{*}\right) \subset \partial_{\mathrm{B}} \mathscr{V}\left(x^{*}\right) \partial_{\mathrm{B}} \mathscr{U}\left(x^{*}\right)$. If for all $F, \tilde{F} \in \partial_{\mathrm{B}}\left|x^{*}\right|$, $\rho(\mathscr{M}(\alpha ; F, \tilde{F}))<1$, then for all $W \in \partial_{\mathrm{B}} \Theta\left(x^{*}\right)$, we have $\rho(W)<1$. We can complete the proof using Proposition 2.

Corollary 1 Let the point $x^{*}$ satisfy $A x^{*}=\left|x^{*}\right|+b$. Let $A \in C^{n \times n}$ be a sparse and positive definite matrix, and $H=\frac{1}{2}\left(A+A^{\mathrm{T}}\right)=G+K$ and $S=\frac{1}{2}\left(A-A^{\mathrm{T}}\right)$ be its Hermitian and skew-Hermitian parts, respectively. Furthermore, $F, \tilde{F} \in \partial_{\mathrm{B}}\left|x^{*}\right|$. We write

$$
\begin{aligned}
& t_{1}(\alpha)=\left\|(\alpha I+S+K)^{-1}(\alpha I-G)\right\|, \\
& t_{2}(\alpha)=\left\|(\alpha I+G)^{-1}(\alpha I-S-K)\right\|,
\end{aligned}
$$

and

$$
\delta=\max \left\{\left\|(\alpha I+G)^{-1} \tilde{F}\right\|,\left\|(\alpha I+S+K)^{-1} F\right\|\right\} .
$$

If $t_{1}(\alpha) t_{2}(\alpha)<1$ and for all $F, \tilde{F} \in \partial_{\mathrm{B}}\left|x^{*}\right|$,

$$
\delta<\frac{2-2 t_{1}(\alpha) t_{2}(\alpha)}{\sqrt{\left(t_{1}(\alpha)-t_{2}(\alpha)\right)^{2}+4}+t_{1}(\alpha)+t_{2}(\alpha)},
$$

then $x^{*}$ is a point of attraction of the nonlinear GHSSlike iterative method.

Proof: By simple calculations we obtain $\mathscr{M}(\alpha ; F, \tilde{F})=(\alpha I+S+K)^{-1}[(\alpha I-G)+F](\alpha I+$ $G)^{-1}[(\alpha I-S-K)+\tilde{F}]=(\alpha I+S+K)^{-1}(\alpha I-G)(\alpha I+$ $G)^{-1}(\alpha I-S-K)+(\alpha I+S+K)^{-1}(\alpha I-G)(\alpha I+$ $G)^{-1} \tilde{F}+(\alpha I+S+K)^{-1} F(\alpha I+G)^{-1}(\alpha I-S-K)+$ $(\alpha I+S+K)^{-1} F(\alpha I+G)^{-1} \tilde{F}$. Hence

$$
\begin{aligned}
\|\mathscr{M}(\alpha ; F, \tilde{F})\| \\
\leqslant\left\|(\alpha I+S+K)^{-1}(\alpha I-G)\right\| \\
\quad \cdot\left\|(\alpha I+G)^{-1}(\alpha I-S-K)\right\| \\
\quad+\left\|(\alpha I+S+K)^{-1}(\alpha I-G)\right\|\left\|(\alpha I+G)^{-1} \tilde{F}\right\| \\
\quad+\left\|(\alpha I+S+K)^{-1} F\right\|\left\|(\alpha I+G)^{-1}(\alpha I-S-K)\right\| \\
\quad+\left\|(\alpha I+S+K)^{-1} F\right\|\left\|(\alpha I+G)^{-1} \tilde{F}\right\| \\
\leqslant t_{1}(\alpha) t_{2}(\alpha)+\delta\left(t_{1}(\alpha)+t_{2}(\alpha)\right)+\delta^{2} .
\end{aligned}
$$

With the help of (9) we obtain

$$
t_{1}(\alpha) t_{2}(\alpha)+\delta\left(t_{1}(\alpha)+t_{2}(\alpha)\right)+\delta^{2}<1 .
$$

Hence we have

$$
\rho(\mathscr{M}(\alpha ; F, \tilde{F})) \leqslant\|\mathscr{M}(\alpha ; F, \tilde{F})\|<1,
$$

which follows from Theorem 2.

\section{NUMERICAL EXAMPLES}

In this section we present a sample of numerical experiments conducted in order to assess the effectiveness of the Picard-GHSS and nonlinear GHSS-like iterative methods. All experiments were performed in MATLAB R2010a, on an Intel Core i5-3210CPU at $2.50 \mathrm{GHz}$ with $4.00 \mathrm{~GB}$ RAM, and terminated when the current residual satisfied

$$
\frac{\left\|A x^{(k)}-\left|x^{(k)}\right|-b\right\|_{2}}{\|b\|}<10^{-6} .
$$

The stopping criterion for the inner iterations of the Picard-GHSS and nonlinear GHSS-like iterative methods was set to be

$$
\frac{\left\|b^{(k)}-A s^{\left(k, l_{k}\right)}\right\|_{2}}{\left\|b^{(k)}\right\|_{2}} \leqslant \eta_{k},
$$

where $b^{(k)}=\left\|x^{(k)}\right\|+b-A x^{(k)}, s^{\left(k, l_{k}\right)}=x^{\left(k, l_{k}\right)}-$ $x^{\left(k, l_{k}-1\right)}, l_{k}$ is the number of the inner iteration steps and $\eta_{k}$ is the prescribed tolerance for controlling the accuracy of the inner iterations at the $k$ th outer iteration. If $\eta_{k}$ is fixed for all $k$, then it is simply denoted by $\eta_{k}$. Here, we take $\eta=0.1$.

The generated test problems are the twodimensional convection-diffusion equation ${ }^{15}$ :

$$
\begin{gathered}
-u_{x x}-u_{y y}+q\left(u_{x}+u_{y}\right)+p u=f(x, y),(x, y) \in \Omega, \\
u(x, y)=0,(x, y) \in \partial \Omega,
\end{gathered}
$$

where $\Omega=(0,1) \times(0,1), \partial \Omega$ is its boundary, $q$ is a positive constant used to measure the magnitude of the diffusive term, and $p$ is a real number. We use the five-point finite difference scheme for the diffusive terms and the central difference scheme for the convective terms. Let $h=1 /(m+1)$ and $\mathrm{Re}=$ $\frac{1}{2} q h$ denote the equidistant step size and the mesh Reynolds number, respectively. Then we obtain a system of linear equations $A x=d$, where $A$ is a matrix of order $n=m^{2}$ of the form

$$
A=T_{x} \otimes I_{m}+I_{m} \otimes T_{y}+p I_{n}=A_{1}+A_{2}+p I_{n},
$$

with

$$
T_{x}=\left(\begin{array}{cccc}
t_{1} & t_{3} & & \\
t_{2} & t_{1} & \ddots & \\
& \ddots & \ddots & t_{3} \\
& & t_{2} & t_{1}
\end{array}\right)_{m \times m},
$$


Table 1 Numerical results for test problems with different values of $m$ and $q(p=2)$.

\begin{tabular}{|c|c|c|c|c|c|}
\hline$q$ & Method & & $m=10$ & $m=20$ & $m=40$ \\
\hline \multirow[t]{12}{*}{0} & \multirow[t]{3}{*}{ PG } & IT & 9 & 10 & 9 \\
\hline & & CPU & 0.0119 & 0.2686 & 7.5407 \\
\hline & & RES & $9.2 \times 10^{-6}$ & $9.6 \times 10^{-6}$ & $8.3 \times 10^{-6}$ \\
\hline & \multirow[t]{3}{*}{ G } & IT & 7 & 7 & 7 \\
\hline & & CPU & 0.0079 & 0.1044 & 3.1403 \\
\hline & & RES & $3.7 \times 10^{-6}$ & $7.3 \times 10^{-6}$ & $5.7 \times 10^{-6}$ \\
\hline & \multirow[t]{3}{*}{$\mathrm{PH}$} & IT & 13 & 13 & 13 \\
\hline & & CPU & 0.0089 & 0.2271 & 9.6186 \\
\hline & & RES & $7.9 \times 10^{-6}$ & $8.3 \times 10^{-6}$ & $4.4 \times 10^{-6}$ \\
\hline & \multirow[t]{3}{*}{$\mathrm{H}$} & IT & 10 & 10 & 10 \\
\hline & & CPU & 0.0093 & 0.1157 & 3.8794 \\
\hline & & RES & $5.3 \times 10^{-6}$ & $9.5 \times 10^{-6}$ & $9.6 \times 10^{-6}$ \\
\hline \multirow[t]{12}{*}{1} & \multirow[t]{3}{*}{ PG } & IT & 10 & 10 & 10 \\
\hline & & CPU & 0.0051 & 0.1486 & 4.5015 \\
\hline & & RES & $9.8 \times 10^{-6}$ & $8.2 \times 10^{-6}$ & $6.0 \times 10^{-6}$ \\
\hline & \multirow[t]{3}{*}{ G } & IT & 7 & 7 & 7 \\
\hline & & CPU & 0.0050 & 0.1201 & 3.2571 \\
\hline & & RES & $6.9 \times 10^{-6}$ & $8.5 \times 10^{-6}$ & $6.1 \times 10^{-6}$ \\
\hline & \multirow[t]{3}{*}{ PH } & IT & 13 & 13 & 13 \\
\hline & & CPU & 0.0107 & 0.1709 & 5.7426 \\
\hline & & RES & $7.9 \times 10^{-6}$ & $7.0 \times 10^{-6}$ & $7.4 \times 10^{-6}$ \\
\hline & \multirow[t]{3}{*}{$\mathrm{H}$} & IT & 10 & 11 & 11 \\
\hline & & CPU & 0.0058 & 0.1413 & 4.7607 \\
\hline & & RES & $9.9 \times 10^{-6}$ & $8.1 \times 10^{-6}$ & $5.3 \times 10^{-6}$ \\
\hline \multirow[t]{12}{*}{10} & \multirow[t]{3}{*}{ PG } & IT & 10 & 10 & 10 \\
\hline & & CPU & 0.0083 & 0.1382 & 4.4619 \\
\hline & & RES & $9.6 \times 10^{-6}$ & $8.4 \times 10^{-6}$ & $9.1 \times 10^{-6}$ \\
\hline & \multirow[t]{3}{*}{ G } & IT & 7 & 7 & 7 \\
\hline & & CPU & 0.0081 & 0.1169 & 3.2828 \\
\hline & & RES & $6.9 \times 10^{-6}$ & $6.2 \times 10^{-6}$ & $5.1 \times 10^{-6}$ \\
\hline & \multirow[t]{3}{*}{$\mathrm{PH}$} & IT & 13 & 14 & 13 \\
\hline & & CPU & 0.0171 & 0.2006 & 5.6773 \\
\hline & & RES & $9.6 \times 10^{-6}$ & $4.7 \times 10^{-6}$ & $4.7 \times 10^{-6}$ \\
\hline & \multirow[t]{3}{*}{$\mathrm{H}$} & IT & 11 & 11 & 11 \\
\hline & & CPU & 0.0083 & 0.1570 & 4.8900 \\
\hline & & RES & $8.5 \times 10^{-6}$ & $8.4 \times 10^{-6}$ & $5.4 \times 10^{-6}$ \\
\hline
\end{tabular}

PG = Picard-GHSS; G = GHSS-like; PH = Picard-HSS; $\mathrm{H}=$ HSS-like

and

$$
T_{y}=\left(\begin{array}{cccc}
0 & t_{3} & & \\
t_{2} & 0 & \ddots & \\
& \ddots & \ddots & t_{3} \\
& & t_{2} & 0
\end{array}\right)_{m \times m},
$$

where $t_{1}=4, t_{2}=-1-\operatorname{Re}, t_{3}=-1+\operatorname{Re}, I_{m}$ and $I_{n}$ are the identity matrices of order $m$ and $n$, respectively, and $\otimes$ means the Kronecker product. In our numerical experiments, the matrix $A$ in (1) is defined by (10) with different values of $q$ and
Table 2 Numerical results for test problems with different values of $m$ and $q(p=2.5)$.

\begin{tabular}{|c|c|c|c|c|c|}
\hline$q$ & Method & & $m=10$ & $m=20$ & $m=40$ \\
\hline \multirow[t]{12}{*}{0} & \multirow[t]{3}{*}{ PG } & IT & 8 & 9 & 9 \\
\hline & & CPU & 0.0049 & 0.2091 & 5.8690 \\
\hline & & RES & $9.3 \times 10^{-6}$ & $2.0 \times 10^{-6}$ & $2.2 \times 10^{-6}$ \\
\hline & \multirow[t]{3}{*}{ G } & IT & 5 & 5 & 5 \\
\hline & & CPU & 0.0032 & 0.1580 & 3.8387 \\
\hline & & RES & $9.9 \times 10^{-6}$ & $7.4 \times 10^{-6}$ & $6.7 \times 10^{-6}$ \\
\hline & \multirow[t]{3}{*}{$\mathrm{PH}$} & IT & 11 & 11 & 12 \\
\hline & & $\mathrm{CPU}$ & 0.0161 & 0.2055 & 7.4478 \\
\hline & & RES & $9.0 \times 10^{-6}$ & $9.4 \times 10^{-6}$ & $4.0 \times 10^{-6}$ \\
\hline & \multirow[t]{3}{*}{$\mathrm{H}$} & IT & 9 & 10 & 10 \\
\hline & & CPU & 0.0083 & 0.1237 & 6.0485 \\
\hline & & RES & $9.2 \times 10^{-6}$ & $8.1 \times 10^{-6}$ & $5.9 \times 10^{-6}$ \\
\hline \multirow[t]{12}{*}{1} & \multirow[t]{3}{*}{ PG } & IT & 8 & 9 & 9 \\
\hline & & CPU & 0.0096 & 0.2115 & 5.8399 \\
\hline & & RES & $9.2 \times 10^{-6}$ & $1.8 \times 10^{-6}$ & $2.0 \times 10^{-6}$ \\
\hline & \multirow[t]{3}{*}{ G } & IT & 5 & 5 & 5 \\
\hline & & $\mathrm{CPU}$ & 0.0040 & 0.1420 & 3.7202 \\
\hline & & RES & $6.9 \times 10^{-6}$ & $8.5 \times 10^{-6}$ & $6.1 \times 10^{-6}$ \\
\hline & \multirow[t]{3}{*}{ PH } & IT & 11 & 12 & 12 \\
\hline & & CPU & 0.0074 & 0.3014 & 7.9885 \\
\hline & & RES & $9.2 \times 10^{-6}$ & $4.1 \times 10^{-6}$ & $4.7 \times 10^{-6}$ \\
\hline & \multirow[t]{3}{*}{$\mathrm{H}$} & IT & 10 & 10 & 10 \\
\hline & & CPU & 0.0070 & 0.2296 & 6.6400 \\
\hline & & RES & $9.1 \times 10^{-6}$ & $6.0 \times 10^{-6}$ & $4.1 \times 10^{-6}$ \\
\hline \multirow[t]{12}{*}{10} & \multirow[t]{3}{*}{ PG } & IT & 9 & 9 & 9 \\
\hline & & CPU & 0.0112 & 0.2203 & 6.1671 \\
\hline & & RES & $7.5 \times 10^{-6}$ & $8.0 \times 10^{-6}$ & $7.5 \times 10^{-6}$ \\
\hline & \multirow[t]{3}{*}{ G } & IT & 6 & 6 & 6 \\
\hline & & CPU & 0.0068 & 0.1450 & 4.1590 \\
\hline & & RES & $8.7 \times 10^{-6}$ & $5.9 \times 10^{-6}$ & $3.0 \times 10^{-6}$ \\
\hline & \multirow[t]{3}{*}{$\mathrm{PH}$} & IT & 12 & 12 & 12 \\
\hline & & CPU & 0.0150 & 0.2429 & 8.0871 \\
\hline & & RES & $7.6 \times 10^{-6}$ & $5.5 \times 10^{-6}$ & $5.9 \times 10^{-6}$ \\
\hline & \multirow[t]{3}{*}{$\mathrm{H}$} & IT & 10 & 10 & 11 \\
\hline & & CPU & 0.0093 & 0.2298 & 6.2374 \\
\hline & & RES & $8.7 \times 10^{-6}$ & $5.7 \times 10^{-6}$ & $5.6 \times 10^{-6}$ \\
\hline
\end{tabular}

$G=\frac{1}{2}\left(A_{1}+A_{1}^{\mathrm{T}}\right) . \quad$ In order to make the hermitian part of $A_{2}+p I_{n}$ positive definite, we take $p=2$ (or 2.5 ), and we take the zero vector as the initial guess, and the right-hand side vector $b$ of (1) is taken in such a way that the vector $x=\left(x_{1}, x_{2}, \ldots, x_{n}\right)$ with $x_{k}=(-1)^{k} i(k=1,2, \ldots, n)$ is the exact solution.

The optimal parameter $\alpha$ employed in each method is experimentally determined such that it results in the least number of iterations. In Table 1 and Table 2, we report the numerical results from the Picard-HSS, the nonlinear HSS-like, the PicardGHSS and the nonlinear GHSS-like iterations for different $p$ and $q$. We also present the elapsed CPU 
time in seconds for the convergence (denoted by $\mathrm{CPU}$ ) and the number of total iteration steps for the convergence. We find that all the methods give approximate solutions of AVEs for all the different matrix dimensions tried, and the nonlinear GHSSlike method is the most efficient method.

\section{CONCLUSIONS}

In this paper, two kinds of GHSS-based iteration methods based on the generalization of the hermitian and skew-hermitian splitting (GHSS) have been described, and the local convergence of the PicardGHSS and nonlinear GHSS-like iterative methods have been given. Numerical tests show that PicardGHSS and nonlinear GHSS-like iterative methods perform better than Picard-HSS and nonlinear HSSlike methods.

Acknowledgements: He is supported by the Science and Technology Foundation of Guizhou Province (Qian ke he Ji Chu [2016]1161); Guizhou Province Natural Science Foundation in China (Qian Jiao He KY [2016]255); the Doctoral Scientific Research Foundation of Zunyi Normal College (BS[2015]09); High-level Innovative Talents of Guizhou Province (Zun Ke He Ren Cai[2017]8). Liu is supported by National Science Foundations of China (71461027); Science and Technology Talent Training Object of Guizhou Province outstanding youth (Qian ke he ren zi [2015]06); Guizhou Province Natural Science Foundation in China (Qian Jiao He KY [2014]295); 2013, 2014 and 2015 Zunyi 15851 Talents Elite Project funding; Zunyi Innovative Talent Team (Zunyi KH(2015)38); Innovative talent team in Guizhou Province(Qian Ke HE Pingtai Rencai[2016]5619). Tian is supported by Guizhou Province Natural Science Foundation in China (Qian Jiao He KY [2015]451); Science and Technology Foundation of Guizhou Province (Qian ke he J zi [2015]2147). The authors would like to thank the first author of Ref. 11 for help to correct the corresponding information.

\section{REFERENCES}

1. Caccetta L, Qu B, Zhou G (2011) A globally and quadratically convergent method for absolute value equations. Comput Optim Appl 48, 45-58.

2. Noor MA, Iqbal J, Noor KI, Al-Said E (2012) On an iterative method for solving absolute value equations. Optim Lett 6, 1027-33.

3. Prokopyev O (2009) On equivalent reformulations for absolute value equations. Comput Optim Appl 44, 363-72.

4. Zhang C, Wei QJ (2009) Global and finite convergence of a generalized Newton method for absolute value equations. $J$ Optim Theor Appl 143, 391-403.
5. Mangasarian OL (2007) Absolute value equation solution via concave minimization. Optim Lett 1, 3-8.

6. Mangasarian OL (2009) A generalized Newton method for absolute value equations. Optim Lett 3, 101-8.

7. Bello Cruz JY, Ferreira OP, Prudente LF (2016) On the global convergence of the inexact semi-smooth Newton method for absolute value equation. Comput Optim Appl 65, 93-108.

8. Li C (2016) A modified generalized Newton method for absolute value equations. J Optim Theor Appl 170, $1-5$.

9. Rohn J (2009) An algorithm for solving the absolute value equations. Electron J Lin Algebra 18, 589-99.

10. Mangasarian OL (2015) A hybrid algorithm for solving the absolute value equation. Optim Lett 9, 1469-74.

11. Gu XM, Huang TZ, Li HB, Wang SF, Li L (2017) Two CSCS-based iteration methods for solving absolute value equations. $J$ Appl Anal Comput 7, 1336-56.

12. Bai ZZ, Golub GH, Ng MK (2003) Hermitian and skew-Hermitian splitting methods for non-Hermitian positive definite linear systems. SIAM J Matrix Anal Appl 24, 603-26.

13. Benzi M (2009) A generalization of the Hermitian and skew-Hermitian splitting iteration. SIAM $J M a-$ trix Anal Appl 31, 360-74.

14. Salkuyeh DK (2014) The Picard-HSS iteration method for absolute value equations. Optim Lett $\mathbf{8}$, 2191-202.

15. Zhang JJ (2015) The relaxed nonlinear PHSS-like iteration method for absolute value equations. Appl Math Comput 265, 266-74.

16. Clarke FH (1990) Optimization and Nonsmooth Analysis, SIAM, Philadelphia, USA.

17. Qi L, Sun J (1993) A nonsmooth version of Newton's method. Math Program 58, 353-67.

18. Pang JS, Qi L (1993) Nonsmooth equations: motivation and algorithms. SIAM J Optim 3, 443-65.

19. Mangasarian OL, Meyer RR (2006) Absolute value equations. Lin Algebra Appl 419, 359-67.

20. Ortega JM, Rheinboldt WC (2000) Iterative Solution of Nonlinear Equations in Several Variables, SIAM, Philadelphia, USA. 